3. P. Montel, Lȩ̧ons sur les familles normales de fonctions analytiques, Paris, 1927.

4. G. Pólya and G. Szegö, Aufgaben und Lehrsaetze aus der Analysis, Berlin, 1925.

5. L. Weisner, Power series, the roots of whose partial sums lie in a sector, Bull. Amer. Math. Soc. vol. 47 (1941) pp. 160-163.

University of Cincinnati

\title{
A NOTE ON SEPARATION AXIOMS AND THEIR APPLICATION IN THE THEORY OF A LOCALLY CONNECTED TOPOLOGICAL SPACE
}

\section{J. W. T. YOUNGS}

In a recent paper $[1]^{1} \mathrm{G}$. E. Albert and the author attempt a comprehensive study of a locally connected (1.8) topological space from the point of view of Peano space theory [2]. Cyclic elements are defined (2.15) and are themselves found to be locally connected and topological (2.29). Moreover, it is shown that under proper and very natural topologization (3.3) the class of all cyclic elements (the hyperspace) becomes a locally connected topological space (3.3 and 3.8). In fact, this hyperspace has no nondegenerate ${ }^{2}$ cyclic elements (3.17).

For the purposes of this note it is the concept of a hereditary class of spaces which is important (4.1). A subclass $\mathfrak{H C}$ of the class $\mathcal{X}$ of all locally connected topological spaces is hereditary if, whenever $X$ is a space of the class $\mathfrak{F}$ : (1) each true cyclic element (2.15) of $X$ is a member of $\mathfrak{H}$; and (2) the hyperspace $X_{h}$ is in $\mathfrak{H C}$. (It should be remembered that the first condition is the one required of a class for it to be cyclicly reducible in the classical Peano space theory.)

The problem is to define small hereditary classes (4.1). In fact, though there is a smallest hereditary class, an intrinsic definition of it is lacking (4.2-4.5).

In this connection the main results are that: (1) the class of all locally connected $T_{0}$-spaces is a hereditary class $(4.10) ;(2)$ the class of all locally connected $T_{1}$-spaces is not a hereditary class (4.1).

It is the purpose of this note: (1) to define, by means of a separation axiom, a new hereditary class; (2) to place this separation axiom

\footnotetext{
Presented to the Society, September 10, 1942; received by the editors August 3, 1942.

1 Numbers in brackets refer to the bibliography; numbers in parentheses to appropriate paragraphs in [1].

${ }^{2} \mathrm{~A}$ set is degenerate if it is vacuous or contains but a single point.
} 
in the spectrum of available separation axioms; (3) to point out the fitting character of the axiom from the point of view of Peano space theory.

First the axiom:

T: If $x \cdot y=0$, then $\bar{x} \cdot \bar{y}$ is degenerate. ${ }^{2}$

(The reader is reminded that in a topological space the closure of a point may contain many points.)

Theorem. The class of all locally connected T-spaces is hereditary.

Proof. Suppose that $X$ is such a space. It is clear that any true cyclic element $M$ of $X$ is a $T$-space. That $M$ is locally connected has been shown elsewhere (2.29).

Consider the hyperspace $X_{h}$ consisting of the totality of cyclic elements of $X$. Let $\xi$ and $\eta$ be any two distinct points of $X_{h}{ }^{3}$

(1) Suppose that $T^{-1}(\xi)$ is nondegenerate. (For a definition of the transformation $T(X)=X_{h}$ see 3.2.) Then there is a true cyclic element $M$ such that $T^{-1}(\xi)=k(M),(2.14)$.

From the definition of the topologization (3.2) and the fact that $M$ is a closed inverse set, it follows that $T(M)$ is closed. Moreover, $\xi \subset T(M)$, therefore $\bar{\xi} \subset T(M)$, and $T^{-1}(\bar{\xi}) \subset M$.

If $M \cdot T^{-1}(\eta)=0$, let $S$ be the component of $X-M$ which contains $T^{-1}(\eta)$. The existence of $S$ is doubtful only in case $T^{-1}(\eta)$ is nondegenerate. But then $T^{-1}(\eta)$ is contained in some true cyclic element and so lies in a component $S$ of $X-M(2.29$ and 2.15). Now $T^{-1}(\eta) \subset \bar{S}=S+F(S)$, and this is an inverse set (3.10 and 3.11). Therefore, $\bar{\eta} \subset T(\bar{S})$, and $T^{-1}(\bar{\eta}) \subset \bar{S}$. Hence, $T^{-1}(\bar{\xi} \cdot \bar{\eta})=T^{-1}(\bar{\xi}) \cdot T^{-1}(\bar{\eta})$ $C M \cdot \bar{S}=F(S)$ which is degenerate (2.15). Therefore $\bar{\xi} \cdot \bar{\eta}$ is degenerate.

If $M \cdot T^{-1}(\eta) \neq 0$, then $T^{-1}(\eta)=y \in M-k(M)$ (2.12 and 2.15). Hence $y$ is a cut point of the space, and as such $\bar{y}=y$ (2.2). Therefore $\bar{\eta}=\eta$ and $\bar{\xi} \cdot \bar{\eta}=\bar{\xi} \cdot \eta$ is certainly degenerate.

(2) A similar argument applies in the event $T^{-1}(\eta)$ is nondegenerate.

(3) If $T^{-1}(\xi)=x$ and $T^{-1}(\eta)=y$, then $\bar{x}$ and $\bar{y}$ are inverse sets (4.9) and so $T(\bar{x})$ and $T(\bar{y})$ are closed in $X_{h}$. Hence these sets contain $\bar{\xi}$ and $\bar{\eta}$, respectively; moreover, $T^{-1}(\bar{\xi}) \subset \bar{x}$ and $T^{-1}(\bar{\eta}) \subset \bar{y}$. Therefore $T^{-1}(\bar{\xi} \cdot \bar{\eta})=T^{-1}(\bar{\xi}) \cdot T^{-1}(\bar{\eta}) \subset \bar{x} \cdot \bar{y}$ which is degenerate because $X$ is a

${ }^{3}$ The reader who is unfamiliar with [1] can still follow the spirit of the argument, if he is acquainted with [2], by thinking of $X$ as a Peano space. In case $T^{-1}(\xi)$ is degenerate think of it as a cut point or an end point. If it is nondegenerate, think of it as a true cyclic element less all the cut points of $X$. As for the topologization in $X_{h}$, a set is open if and only if its inverse under $T$ is open in $X$. 
$T$-space. This implies $\bar{\xi} \cdot \bar{\eta}$ is degenerate, and the proof is complete on observing that $X_{h}$ is locally connected and topological (3.3 and 3.8).

The separation axioms mentioned in the course of this note may be tabulated as follows:

$T_{0}: \quad x \cdot \bar{y}+\bar{x} \cdot y$ is degenerate.

$T: \bar{x} \cdot \bar{y}$ is degenerate.

If $x \cdot y=0$, then

$$
\begin{array}{ll}
T_{1}: & x \cdot \bar{y}+\bar{x} \cdot y=0 . \\
T_{1}: & \bar{x} \cdot \bar{y}=0 \text { (alternate form). }
\end{array}
$$

Theorem. $A T_{1}$-space is a $T$-space; a $T$-space is a $T_{0}$-space.

The proof is left as an entertaining exercise for the reader. An immediate consequence is that the $T$-axiom might very well have attached to the letter $T$ as a subscript any positive number less than one.

In conclusion it might be interesting to note that the $T$-axiom fits the phenomenon of Peano space theory relative to intersections of cyclic elements. The theorem that two cyclic elements which have more than one point in common are identical has as its counterpart the statement that two points whose closures have more than one point in common are identical.

\section{BIBLIOGRAPHY}

1. G. E. Albert and J. W. T. Youngs, The structure of locally connected topological spaces, Trans. Amer. Math. Soc. vol. 51 (1942) p. 637.

2. C. Kuratowski and G. T. Whyburn, Sur les élêments cycliques et leurs applications, Fund. Math. vol. 16 (1930) p. 305.

PuRdue University 